\title{
Increased Risk of Childhood Brain Tumors among Children Whose Parents had Farm-Related Pesticide Exposures during Pregnancy
}

\author{
B. Kunkle ${ }^{\mathrm{a}}$, K.P. Singh ${ }^{\mathrm{b}}$, and D. Roy \\ ${ }^{a}$ Department of Environmental and Occupational Health, Florida International University, Miami, Florida \\ 33199; ${ }^{b}$ Division of Preventive Medicine, University of Alabama at Birmingham, Birmingham, AL 35205 \\ Email:Droy@fiu.edu
}

\begin{abstract}
Malignant brain tumors rank second in both incidence and mortality by cancer in children, and they are the leading cause of cancer death in children. Relatively little is known about the etiology of childhood brain tumor (CBT). While there are several studies which link pesticide exposure to increased risk of CBT, findings have been inconsistent. We performed a meta-analysis on 15 published epidemiological studies to test that in utero exposure to pesticides may be involved in the development of brain cancer in children. Meta-analysis was performed using the general variance-based method and homogeneity was tested by means of the Q statistic. Summary relative risk (RR) estimates were calculated for childhood brain cancer from 1) paternal exposure to pesticides prior to conception, 2) both maternal and paternal exposure to pesticides during pregnancy, 3) maternal exposure during pregnancy to: a. agricultural and $\mathrm{b}$. non-agricultural activities, and 4) childhood exposure to: a. agricultural and b. nonagricultural activities up to date of diagnosis with CBT. The Comparative Toxicogenomics Database (CTD) was used to identify gene-pesticideCBT interactions. Findings of meta-analyses revealed a significantly increased risk of CBT among children whose mothers had farm-related exposures during pregnancy $(\mathrm{RR}=1.48,95 \% \mathrm{CI}=1.18-1.84)$. A dose response was recognized when this risk estimate was compared to those for risk of CBT from maternal exposure to non-agricultural pesticides (e.g. home extermination, pest strips) during pregnancy ( $R R=1.36$, 1.10-1.68), and risk of CBT among children exposed to agricultural activities ( $R R=1.32,1.04-1.67)$. Three studies combined for the paternal exposure to pesticides during preconception produced a calculated summary risk estimate of odds ratio $(\mathrm{OR})=2.29(95 \% \mathrm{CI}: 1.39-3.78)$. Meta-analysis of five studies of paternal exposure to pesticides during pregnancy produced a final calculated summary risk estimate of OR $=$ 1.63 (95\% CI: $1.16-2.31)$. The search of the CTD databases revealed association between herbicide and astrocytoma and more than 300 genes are altered by exposure to herbicide, fungicide, insecticide or pesticides. In summary, comparing results from our categories of exposure, pre-conception and pregnancy exposure estimates were slightly higher than childhood exposure estimates, paternal exposures produced slightly higher risk estimates compared to maternal exposures, agricultural exposures produced slightly higher risk estimates compared to non-agricultural exposures and CTD search revealed potential genespesticides-astrocytoma interactions. Based on the collective results of these meta-analyses it appears that pesticide exposure may increase risk of CBT, with preconception and prenatal exposures being especially important factors in increasing risk of its development. Interestingly, paternal exposure may be as important, if not more important than maternal exposures, particularly during the preconception period. Whether this is a result of paternal exposures being more prevalent than maternal exposures or the consequence of a biological process, is a question that deserves further attention in future investigations of CBT etiology.
\end{abstract}

Keywords: $\quad$ Childhood brain tumor (CBT), Pesticide exposure, Increased risk 


\section{INTRODUCTION}

Links between pesticide exposure and childhood tumors were first noted in case reports in the 1970s [1-3]. Since then, numerous studies have been published that support a role for pesticides in childhood brain tumor (CBT) etiology. To date, three separate reviews of pesticide exposure and CBTs have been published [4-6]. In 1997, Daniels et al. found that the studies to date showed that 1) farm residence was associated with CBT and 2) pesticide use in the home was fairly consistently associated with CBT despite the small sample sizes of studies [4]. However, they concluded that "it remains unclear whether a specific time window of exposure may be of greater importance in studying the effects of home pesticide use." Zahm and Ward's 1998 review noted that nine of the 17 studies through 1998 had reported increased risk of CBT from pesticide exposure, five additional studies found a positive relationship that was not statistically significant, and only three studies reported no excess risk associated with pesticide exposure [6]. Finally, Jurewics and Hanke 2006 reported that most studies since 1998 found associations between CBT and both agricultural employment and home use of pesticides [5]. While occupational exposure is an established pathway for significant pesticide exposure, contact through home use of pesticides has been of less concern. However, substantial pesticide exposure to children can occur in and around the home, with those being used outdoors being tracked into the home on shoes and by pets. $82 \%$ of United States households use pesticides with an average of 3 to 4 different pesticide products being used per home [7]. These exposures can become long-lasting as the pesticide residues can remain in carpets, furniture, and toys without being degraded by processes that exist outdoors (e.g., rain and sun) [6]. Furthermore, Whyatt et al. 2003 examined pesticide levels in plasma of 230 NYC mothers and infant pairs, finding that 7 pesticides were detected in up to $83 \%$ of the plasma sample [8]. Maternal plasma levels were correlated with cord blood levels, indicating placental transfer from pregnant mother to fetus [9]. However, most studies on the relation between pesticide exposure and CBT have used small sample sizes and to date no meta-analysis of these studies has been published. While not a pediatric study, a meta-analysis of adult BT and farming did find an increased RR of 1.30 (95\% CI: $1.09-1.56)$ [10]. By conducting a meta-analysis of the studies on CBT and pesticides we aim to synthesize past information on this topic and produce more robust risk estimates of any relations that may exist. We have analyzed the importance of maternal and paternal exposures in CBT etiology, the difference in CBT risk for agricultural and non-agricultural pesticides exposures, and searched for genes that interact with pesticides and have their expression linked to the development of astrocytoma using toxicogenomics data base.

\section{METHODS}

A PubMed search was conducted to identify studies of the association between CBT and pesticides. This search included articles published from 1966 until December 17, 2010. Search terms used in various combinations were brain, cancer, tumor, childhood, adolescent, in utero, pregnancy, central nervous system, pesticides, farming, and agriculture. References cited in the studies identified by this search were also reviewed for inclusion in the meta-analyses. Inclusion criteria for initial search were: 1) Published in English language peer-reviewed journals between 1966 and 2006; 2) Provided sufficient data to determine an estimator of relative risk (RR) for CBT and its confidence interval (CI); and, 3) Study evaluated pesticide and/or farm exposure and CBT. Exclusion criteria for initial search were: 1) Did not report original results (reviews, comments, letters, etc.); 2) Results already reported in another study or in a more comprehensive study; 3) Geographic studies using GIS, etc.; 4) Study had less than 4 cases in subgroup of interest; and, 5) Study did not report timing of exposure. For timing of exposure a study was excluded if the exposure period was not specific to one of our identified time periods. For example, Kristensen et al. 1997 used a 5 year period of exposure and was thus excluded from the final analyses [10]. CBT was defined as any child diagnosed with CBT until age 19. This conformed with the standard SEER (Surveillance, Epidemiology, and End Results) childhood cancer classification. CBT included all central nervous system tumors including brain tumors such as astrocytoma and primitive neuroectodermal tumor (PNET) and other central nervous system tumors such as neuroblastoma. Pesticide exposures were defined as either agricultural or nonagricultural in nature. Agricultural pesticide exposures were defined as exposures to pesticides from farming, farm related activities, living on a farm, mixing and preparing agricultural pesticides, horticulture, nursery work, and professional gardening. Non-agricultural pesticide exposures were those that occurred during home use such as flea/tick pesticide use, garden pesticide use, yard treatment, pest strip use, and home extermination. The data were stratified three separate ways and several separate meta-analyses were performed among these groups. Stratification methods included 1) exposure categories separated into maternal exposures, paternal exposures, and child exposures; 2) exposure time period separated into preconception, pregnancy, and childhood; and 3) type of exposure split into agricultural pesticide exposures and non-agricultural pesticides exposures. A general variance-based method using confidence intervals 
developed independently by Prentice and Thomas [11] and Greenland [12] was used to calculate relative risk estimates. This method requires only information on each study's estimate of relative risk and its $95 \%$ confidence interval, reducing the amount of studies excluded from the analysis because of missing data. The weight given to each study is chosen to be the inverse of the variance of the effect estimate, meaning that larger studies with smaller standard errors are given more weight than smaller studies that have larger standard errors. In addition, because adjusted rate ratios and confidence intervals are used in the analysis, the method takes confounding into consideration, unlike other meta-analyses methods which may not include confounding in their estimates. Analysis was completed using Comprehensive Meta Analysis Version 2.2.046 from Biostat, Inc. which can be downloaded at www.Meta-Analysis.com [13]. Risk estimates were combined following several pre-determined rules. For studies that reported risk estimates for different types of CBT separately without reporting a summary estimate for all CBT, we calculated a summary risk estimate for use in our meta-analyses. In addition, when a study reported more than one risk estimate for different pesticide exposure types or situations, the general exposure category was used for the meta-analyses calculations. Finally, the studies that reported home exposure/use of pesticides during pregnancy without specifying it as either maternal or paternal specifically were included in the maternal exposure during pregnancy category. This was based on the concept that the mother is the ultimate exposure during this period.

We used the Comparative Toxicogenomics Database (CTD) located at: http://www.mdibl.org/research/ctd.shtml [14] to investigate the association between pesticides and astrocytoma or genes-pesticide interactions. We searched astrocytoma to obtain a list of genes found to be modulated by pesticide exposures in astrocytoma. To perform our search we entered 'astrocytoma' into a disease search, and then chose the 'chemicals' or 'chemical-gene interactions' tab. The second search was conducted by selecting 'chemicals' and followed by chosing the 'pesticides' and or 'chemical-gene interactions' tab.

\section{RESULTS}

The initial literature search produced 33 studies that fit the inclusion criteria. 16 studies were rejected by the exclusion criteria, leaving 17 studies for the final meta-analyses calculations. Results of the meta-analyses performed can be found in table 1 .

Table 1. Summary of results of metaanalyses of the association between pesticides and childhood brain tumors.

\begin{tabular}{|c|c|c|c|}
\hline Time Period & Exposure Category & $\begin{array}{l}\text { Number of Studies in } \\
\text { Estimate }\end{array}$ & $\begin{array}{l}\text { Summary Risk Estimate } \\
\left(95^{\circ} \text { Confidence Interval) }\right.\end{array}$ \\
\hline \multirow{2}{*}{ Pre-conception } & Matemal Exposures & 1 & $0.87(0.29 \cdot 2.60)$ \\
\hline & \begin{tabular}{|l|l|} 
Patemal Exposures \\
\end{tabular} & 3 & $2.29(1.39-3.78)$ \\
\hline \multirow{3}{*}{ Pregnancy } & Matemal Exposures(Agicultural) & 5 & $1.48(1.18 \cdot 1.84)$ \\
\hline & \begin{tabular}{|l} 
Matemal Exposures (Non-agicultural) \\
\end{tabular} & 7 & $1.36(1.10 \cdot 1.68)$ \\
\hline & \begin{tabular}{|l|l|} 
Patemal Exposures \\
\end{tabular} & 5 & $1.63(1.16 \cdot 2.31)$ \\
\hline \multirow{2}{*}{ Childhood } & \begin{tabular}{|l} 
Agricultural Exposures \\
\end{tabular} & 4 & $1.35(1.08 \cdot 1.70)$ \\
\hline & \begin{tabular}{|l|l|l|l|} 
Non-aggicultural Exposures \\
\end{tabular} & 5 & $1.32(1.04-1.67)$ \\
\hline
\end{tabular}

\section{Exposure during Pre-conception}

No meta-analysis was performed on maternal exposure to pesticides during preconception as only one study fit the criteria. Three studies were combined for the paternal exposure to pesticides during preconception, producing a calculated summary risk estimate of 2.29 (95\% CI: $1.39-3.78$ ) (Table 2). 
Exposures during Pregnancy

All three summary risk estimates for exposure during pregnancy showed a significant association between pesticide exposure and CBT. A combination of 5 studies for maternal exposure to agricultural pesticides during pregnancy generated an increased risk of 1.48 (95\% CI: $1.18-1.84)$ (Table 3). Seven studies of maternal exposure to non-agricultural pesticides during pregnancy produced a summary risk estimate of 1.36 (95\% CI: 1.10 - 1.68) (Table 4). Finally, the paternal exposure to pesticides during pregnancy meta-analysis grouped five studies for a final calculated risk estimate of 1.63 (95\% CI: $1.16-2.31)$ (Table 5).
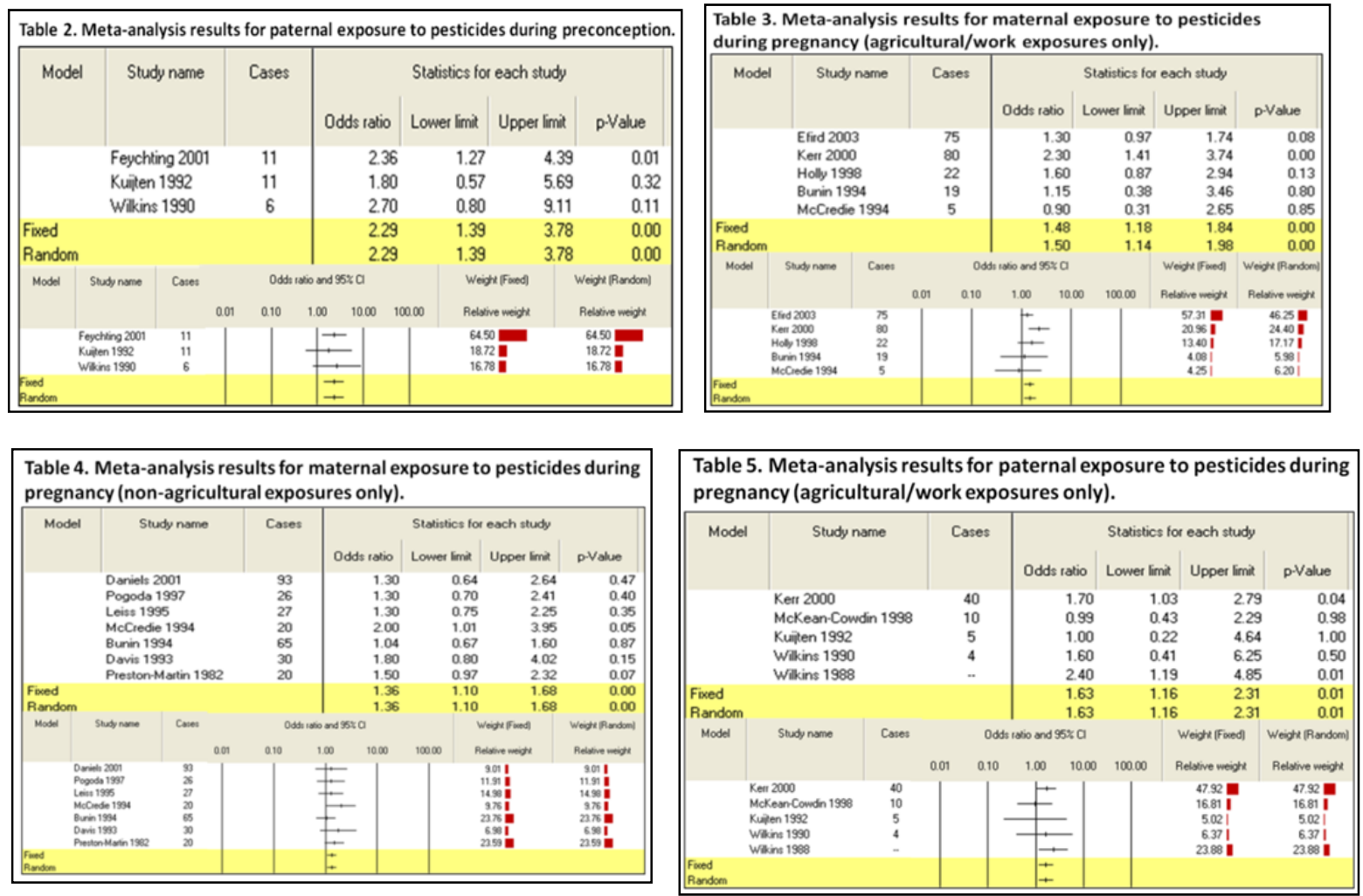

Exposures during Childhood

Combining four studies of childhood exposure to agricultural pesticides produced a summary risk estimate of 1.35 (95\% CI: 1.08 - 1.70) (Table 6). Non-agricultural pesticide exposures during childhood were also found to be significantly associated with development of CBT as a meta-analysis of five studies found an increased risk of 1.32 (95\% CI: $1.04-1.67)$ (Table 7).
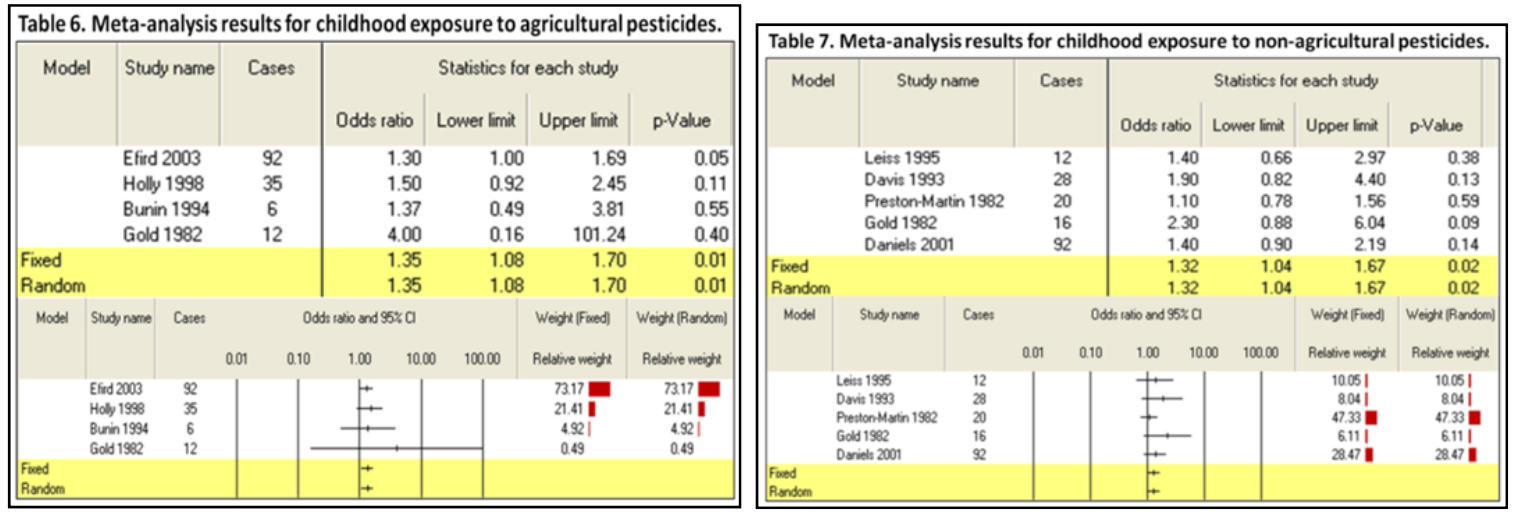

The search of the CTD databases revealed association between herbicide and astrocytoma [15]. The CTD search revealed that more than 300 genes are altered by exposure to herbicide, fungicide, insecticide or pesticides. For example, herbicide results in increased expression of several genes, such as A2M, ABCB4 as well as decreased expression of many genes, ADH8, AGXT2, AGMO. Fungicide, fluconazole results in decreased expression of ABCB9 mRNA. Insecticides results in decreased expression of ACACA mRNA [16]. Pesticides results in decreased activity of ACHE protein [17,18]. 


\section{DISCUSSION AND CONCLUSIONS}

Our meta-analyses produced several estimates of increased risk of CBT from exposure to pesticides. Comparing results from our categories of exposure, pre-conception and pregnancy exposure estimates were slightly higher than childhood exposure estimates, paternal exposures produced slightly higher risk estimates compared to maternal exposures, and agricultural exposures produced slightly higher risk estimates compared to non-agricultural exposures.

Evidence supporting a possible role for paternal exposure to pesticides during preconception and increased risk of CBT is limited but does exist. Tomatis et al. 1981 reported male rats given a single dose of ethylnitrosourea prior to mating with untreated females produced offspring at increased risk for CNS tumors (5.6\% compared to $1.9 \%$ controls; $p=0.08)$ [19]. Yu et al. 1999 found that offspring of male mice treated with chromium two weeks before conception were at increased risk of tumors. This study also reported that methylation changes were noted in the sperm and could have altered parental imprinting [20]. Lastly, a population study by Infante-Rivard et al. 1999 found increased risk of leukemia in children whose fathers were exposed to pesticides during preconception [21].

There are several strengths of this meta-analysis. As mentioned earlier, the use of the general variance based method gave more weight to larger studies, considered confounding, and limited the number of studies excluded because of missing data. Most studies used interview data to assess exposure, providing a more direct accounting of exposure. Finally, the combining of similar exposure time periods and splitting of maternal/paternal and agricultural/non-agricultural exposures allowed for assessment of the range of possible external etiological factors involved in CBT development.

Limitations of the study include those typical of the epidemiological studies combined in meta-analyses such as publication bias, recall bias and exposure misclassification. Also, pesticide and CBT type, along with individual practices of participants, were not distinguished in most studies. Lastly, a few studies had correlations for exposures across three time periods, thus limiting our ability to evaluate fully the independent effects of pesticide exposure during specific time periods.

Based on the collective results of these meta-analyses it appears pesticide exposure does increase risk of CBT, with preconception and prenatal exposures being especially important factors in increasing risk of its development. Interestingly, paternal exposure may be as important, if not more important than maternal exposures, particularly during the preconception period. Whether this is a result of paternal exposures being more prevalent than maternal exposures or the consequence of a biological process, is a question that deserves further attention in future investigations of CBT etiology. The need for CBT exposure studies with better exposure time period characterization, including the division of pregnancy exposures into trimester, is also apparent.

\section{REFERENCES}

1. Infante,P.F. and Newton,W.A., Jr. (1975) Letter: Prenatal chlordane exposure and neuroblastoma. N.Engl.J.Med., 293, 308.

2. Infante,P.F. and Freeman,C. (1987) Cancer mortality among workers exposed to chlordane. J.Occup.Med., 29, 908-911.

3. Pratt,C.B., Rivera,G., Shanks,E., Johnson,W.W., Howarth,C., Terrell,W., and Kumar,A.P. (1977) Colorectal carcinoma in adolescents implications regarding etiology. Cancer, 40, 24642472.

4. Daniels,J.L., Olshan,A.F., and Savitz,D.A. (1997) Pesticides and childhood cancers. Environ.Health Perspect., 105, 1068-1077.

5. Jurewicz,J., Hanke,W., Johansson,C., Lundqvist,C., Ceccatelli,S., van den Hazel,P., Saunders,M., and Zetterstrom,R. (2006) Adverse health effects of children's exposure to pesticides: what do we really know and what can be done about it. Acta Paediatr.Suppl, 95, 7180.

6. Zahm,S.H. and Ward,M.H. (1998) Pesticides and childhood cancer. Environ.Health Perspect., 106 Suppl 3, 893-908.

7. Khuder,S.A., Mutgi,A.B., and Schaub,E.A. (1998) Meta-analyses of brain cancer and farming. Am.J.Ind.Med., 34, 252-260. 
8. Whyatt,R.M., Barr,D.B., Camann,D.E., Kinney,P.L., Barr,J.R., Andrews,H.F., Hoepner,L.A., Garfinkel,R., Hazi,Y., Reyes,A., Ramirez,J., Cosme,Y., and Perera,F.P. (2003) Contemporaryuse pesticides in personal air samples during pregnancy and blood samples at delivery among urban minority mothers and newborns. Environ.Health Perspect., 111, 749-756.

9. U.S.Environmental Protection Agency. US EPA National Home and Garden Pesticide Use Survey. 1990. Washington, DC.

10. Kristensen,P., Irgens,L.M., Andersen,A., Bye,A.S., and Sundheim,L. (1997) Birth defects among offspring of Norwegian farmers, 1967-1991. Epidemiology, 8, 537-544.

11. Prentice,R.L. and Thomas,D.B. (1987) On the epidemiology of oral contraceptives and disease. Adv.Cancer Res., 49, 285-401.

12. Greenland,S., Schlesselman,J.J., and Criqui,M.H. (1986) The fallacy of employing standardized regression coefficients and correlations as measures of effect. Am.J.Epidemiol., 123, 203-208.

13. Borenstein M, Hedges L, Higgins J, and Rothstein H. Comprehensive Meta-analysis Version 2. 2005. Englewood NJ, Biostat.

14. Davis AP, Murphy CG, Saraceni-Richards CA, Rosenstein MC, Wiegers TC, Mattingly CJ. Comparative Toxicogenomics Database: a knowledgebase and discovery tool for chemicalgene-disease networks. Nucleic Acids Res 2009; 37:D786-D792.

15. Shim YK, Mlynarek SP, van Wijngaarden E..Parental exposure to pesticides and childhood brain cancer: U.S. Atlantic coast childhood brain cancer study. Environ Health Perspect. 2009 Jun;117(6):1002-6.

16. Evrard E, Marchand J, Theron M, Pichavant-Rafini K, Durand G, Quiniou L, Laroche J.. Impacts of mixtures of herbicides on molecular and physiological responses of the European flounder Platichthys flesus. Comp Biochem Physiol C Toxicol Pharmacol. 2010 Sep;152(3):321-31

17. Kuang XY, Zhou ZJ, Ma XX, Yao F, Wu QE, Chen B..Activity of esterases and effect of genetic polymorphism in workers exposed to organophosphorus pesticides.Zhonghua Lao Dong Wei Sheng Zhi Ye Bing Za Zhi. 2006 Jun;24(6):333-6.

18. Hernández AF, López O, Rodrigo L, Gil F, Pena G, Serrano JL, Parrón T, Alvarez JC, Lorente JA, Pla A..Changes in erythrocyte enzymes in humans long-term exposed to pesticides: influence of several markers of individual susceptibility. Toxicol Lett. 2005 Oct 15;159(1):1321.

19. Tomatis,L., Cabral,J.R., Likhachev,A.J., and Ponomarkov,V. (1981) Increased cancer incidence in the progeny of male rats exposed to ethylnitrosourea before mating. Int.J Cancer, 28, 475478.

20. Yu,W., Sipowicz,M.A., Haines,D.C., Birely,L., Diwan,B.A., Riggs,C.W., Kasprzak,K.S., and Anderson,L.M. (1999) Preconception urethane or chromium(III) treatment of male mice: multiple neoplastic and non-neoplastic changes in offspring. Toxicol.Appl.Pharmacol., 158, 161-176.

21. Infante-Rivard,C., Labuda,D., Krajinovic,M., and Sinnett,D. (1999) Risk of childhood leukemia associated with exposure to pesticides and with gene polymorphisms. Epidemiology, 10, 481-487. 